\title{
Shear-Induced Long-Range Uniaxial Assembly of Polyaromatic Monolayers at Molecular Resolution
}

\author{
Shern-Long Lee, ${ }^{\dagger}$ Cher-Yi Jason Chi, ${ }^{\dagger}$ Min-Jie Huang, ${ }^{\dagger}$ Chun-hsien Chen, ${ }^{*}{ }^{\dagger}$ Chia-Wen Li, ${ }^{\ddagger}$ \\ Kamalkishore Pati, ${ }^{\ddagger}$ and Rai-Shung Liu $^{\ddagger}$ \\ Department of Chemistry, National Taiwan University, Taipei, Taiwan 10617, and Department of Chemistry, \\ National Tsing Hua University, Hsinchu, Taiwan 30043
}

Received April 2, 2008; E-mail: chchen@ntu.edu.tw

(WI) This paper contains enhanced objects available on the Internet at http://pubs.acs.org/jacs.

The control of spatial arrangements of molecular building blocks on surfaces opens the foundational step of the bottom-up approach toward future nanotechnologies. ${ }^{1,2}$ Molecular self-assembly seems to be an effortless strategy to shape supramolecular architectures via the spontaneous association of tailored molecular constituents which, however, develop a complex balance of intramolecular, intermolecular, and molecule-substrate interactions. ${ }^{1}$ The interplay of these weak and subtle interactions is difficult to harness and consequently results in polycrystallinity or amorphism at the submicrometer level. Only very recently, well-defined stacking of porphyrin trimers was periodically patterned up to $1 \mathrm{~mm}$ at a solid/liquid interface by Elemans and coworkers who ingeniously had the macroscopic dewetting of solvent guide the alignment of porphyrin macrocycles. ${ }^{3}$ A close resemblance to such a hierarchical control in which the forces at the macroscopic level govern over those on the nanometer scale can be found in Couette flow, ${ }^{4}$ namely, the laminar flow between two concentric walls, one of which rotates and creates viscous shear proven useful to align macromolecules. ${ }^{5}$ Although liquid crystals can be oriented to a high degree by simple shear, ${ }^{6,7}$ examples of millimeter-scale uniaxially aligned monolayers of small molecules at solid surfaces are still unprecedented. Here we report the assembly of polyaromatic monolayers that exhibit predominantly one single-domain up to $7 \mathrm{~mm}$ by planar Couette-like flow ${ }^{4}$ whose anisotropic shear induces an edgeon orientation for alkoxylated dibenzo $[g, p]$ chrysene $^{8}$ (DBC), which would otherwise adopt the face-on arrangement on graphite surface.

To acquire the uniaxially aligned features, the films were prepared on atomically smooth highly orientated pyrolytic graphite (HOPG) and characterized by scanning tunneling microscopy (STM). The effect of the Couette-like shear on the orientation of DBC in phenyloctane is demonstrated in Figure 1 where the films in panels a and $b$ were, respectively, with and without being subjected to the shear treatment (vide infra). The symmetries of the adlayers were distinctly different. Without the shear (Figure 1b) DBC adopted a face-on orientation, characteristics of polyaromatics on HOPG. ${ }^{9,10}$ Remarkably, the shear treatment induced a transition from a face-on to an edge-on orientation and drove the molecules stacked along the [0110] direction of HOPG, judged by the change in the adlattice symmetry and by the decrease of the nearest neighbor spacing from 1.7 to $0.5 \mathrm{~nm}$. To show the generality of this method, displayed in Figure S1 (Supporting Information) are images of the shear-treated films of alkylated tetrabenz $[a-$ $, c, h, j]$ anthracene and hexa-peri-hexabenzocoronene, representing molecules of rectangular and discotic shapes, respectively. Also shown in Figure S1 is coronene, representing unsubstituted polyaromatic hydrocarbons. After the Couette-like shear treatment, stacking features appeared and they generally aligned continuously across local defects,

\footnotetext{
$\uparrow$ National Taiwan University.

* National Tsing Hua University.
}

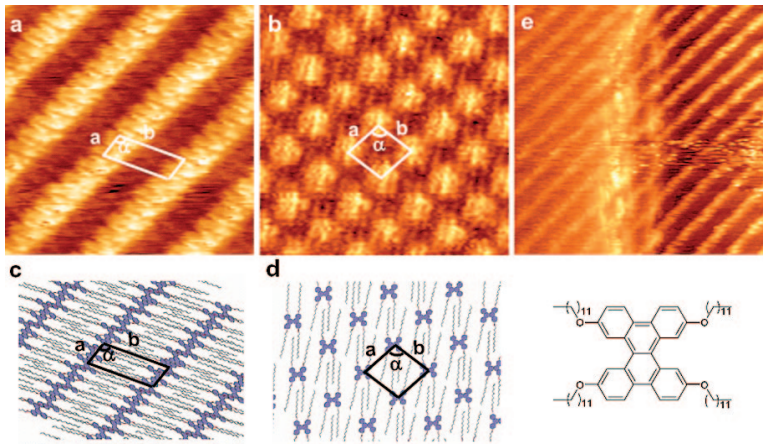

Figure 1. Adlattice structures of 3,6,11,14-tetradodecyloxydibenzo[g,p]chrysene for films (a) being subjected to the shear treatment sketched in Figure 2 and (b) prepared by drop cast. (c and d) Proposed adlattices. (e) Face-to-face molecular packing extending across local defects. $|\vec{a}|,|\vec{b}|$, and $\alpha$ for images a and $\mathrm{b}$ are, respectively, $1.04 \pm 0.09 \mathrm{~nm}, 3.06 \pm 0.13 \mathrm{~nm}, 115^{\circ} \pm 3^{\circ}$, and 1.70 $\pm 0.11 \mathrm{~nm}, 1.92 \pm 0.12 \mathrm{~nm}, 95^{\circ} \pm 4^{\circ}$. Note that in panels a and $\mathrm{c}$ there are two molecules per unit cell because the molecules are zigzagged along $\overrightarrow{\mathrm{a}}$, parallel to $[01 \overline{1} 0]$ of the underlying HOPG (lattice is not drawn for clarity). DBC was dissolved and imaged in phenyloctane. The $E_{\text {bias }}, I_{\text {tunneling, }}$ and imaging sizes for images a, b, and e, are, respectively, $-0.80 \mathrm{~V}, 60 \mathrm{pA}, 12 \times 12 \mathrm{~nm}^{2},-0.85$ $\mathrm{V}, 36 \mathrm{pA}, 12 \times 12 \mathrm{~nm}^{2}$, and $-0.80 \mathrm{~V}, 60 \mathrm{pA}, 30 \times 30 \mathrm{~nm}^{2}$.

such as step edges (e.g., Figure 1e), indicative of fairly strong intermolecular interactions.

Typical images of the uniaxially stripe-patterned monolayers are presented in Figure 2. The $0.2-\mu \mathrm{m}$ scan size of images $b_{1}-b_{5}$ approaches the limit allowing the individual stripes to be discernible and simultaneously demonstrates the degree of the long-range order across the connected images. Locations $\mathrm{a}$ and $\mathrm{b}$ were spaced apart by about $7 \mathrm{~mm}$ yet both exhibited streaks propagated along the same direction (Figure S2).

Two approaches were designed to easily prepare extraordinary long stacks of polyaromatics. The shear flow was generated by a peristaltic pump (method 1) or by the capillary force from a piece of lens paper (method 2).

A movie, video 1, presenting the ease of method 1 is provided.

Briefly, a drop of sample was placed on HOPG over which a glass slide was glided by a syringe pump or a peristaltic pump. STM revealed that the molecules faced on at a slow speed $(\leq 0.2 \mathrm{~mm} / \mathrm{s})$ and became stacked stripes when the glass slide moved faster than $8 \mathrm{~mm} / \mathrm{s}$, where the respective rates corresponded to the maximal speed of the syringe pump and the minimum of the peristaltic pump. The dependence of the molecular orientation on the moving speed of the glass slide manifests that certain strength of the shear at the liquid/solid interface was required to agitate the adsorbed molecules and to prompt the faceto-face packing arrangement. However, the stripes appeared wavy 


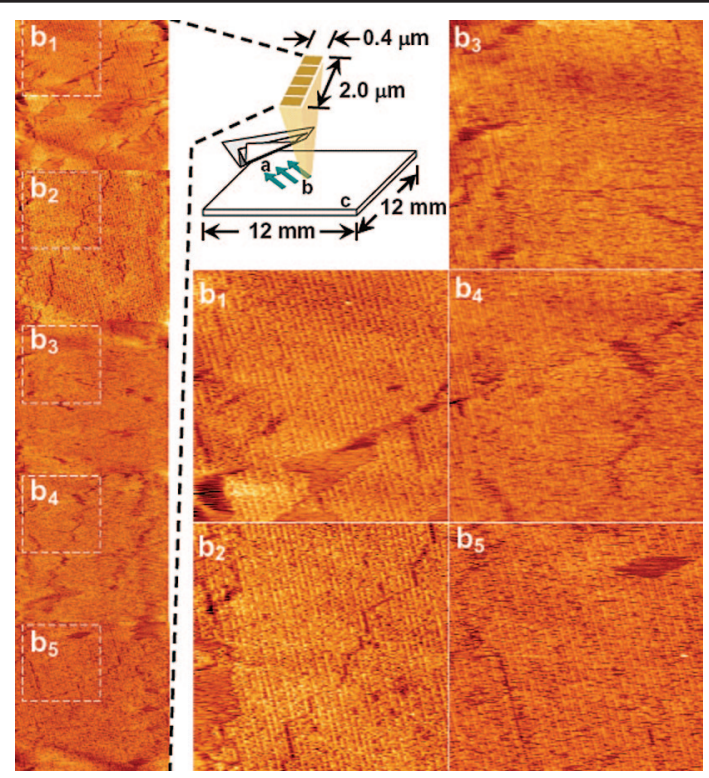

Figure 2. Scheme illustrating how the monolayers were aligned and indicating where the STM images were acquired for monolayers of DBC in phenyloctane. The lens paper was placed at the corner and vertical to [01 10$]$ of HOPG. The blue arrows indicate the flow direction of the sample solution. Locations $a, b$, and $\mathrm{c}$ were, respectively, near the origin where the sample solution was blotted, at the center of the HOPG (ca. $\sim 7 \mathrm{~mm}$ from the corner), and at the opposite corner from location a. The five frames on the left were obtained from location $\mathrm{b}$. The dashed squares of $\mathrm{b}_{1}-\mathrm{b}_{5}$ were magnified $\left(200 \times 200 \mathrm{~nm}^{2}, E_{\text {bias }},-0.85\right.$ $\mathrm{V} ; I_{\text {tunneling }}, 36 \mathrm{pA}$ ) to show that the striped patterns span for at least a $2-\mu \mathrm{m}$ distance. The images at locations a and $\mathrm{c}$ were similar to those of $\mathrm{b}_{1}-\mathrm{b}_{5}$ (stacked molecules) and Figure $1 \mathrm{~b}$ (face on), respectively, and thus were not shown.

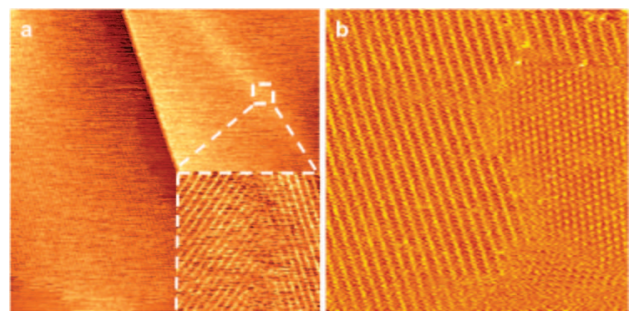

Figure 3. STM image exhibiting multidomains for DBC films. (a) The film was prepared when the shear was $\sim 30^{\circ}$ off from [0110] of HOPG. (b) Coexistence of edge-on and face-on orientations of DBC near location c in Figure 2. Conditions: $E_{\text {bias }},-0.80 \mathrm{~V} ; I_{\text {tunneling }}, 60 \mathrm{pA}$. Image size: (a) $350 \times$ $350 \mathrm{~nm}^{2}, 50 \times 50 \mathrm{~nm}^{2}$ (inset), (b) $40 \times 40 \mathrm{~nm}^{2}$.

(Figure S3). Because the mechanical movement of the glass slide was not perfectly smooth, linear, and parallel to the HOPG substrate, the flow likely carried pulsating unevenness and resulted in stacking faults here and there. Alternatively, after placing a drop of the sample solution on HOPG, a piece of cellulose paper, lens tissue, or Kimwipe (Figure 2) was employed to absorb, to pull the solvent, and thus to drag a shear at the surface for $8-10 \mathrm{~s}$. The linear flow rate was ca. $0.5-0.6$ $\mathrm{mm} / \mathrm{s}^{11}$ This procedure furnished the laminar flow with great steadiness. The striped arrays (e.g., Figure 2) were better aligned and less interrupted than those prepared by method 1 .

To obtain a predominantly single domain assembly on the millimeter scale, it was crucial to steer the solution flow direction parallel to that of the unit cell vectors of HOPG which were predetermined by STM. For example, displayed in Figure 3a was a film subjected to a shear deliberately not parallel to [0110] of HOPG. The angle of ca. $60^{\circ}$ between the domain patches accommodates the symmetry of HOPG and suggests a significant molecule-substrate interaction.
The effect of solvent dewetting is a significant factor on the thinfilm assembly. ${ }^{12-15}$ For films prepared as sketched in Figure 2, the dewetting of the solvent disturbed the span of the aligned arrays. For example, Figure $3 \mathrm{~b}$ was obtained near location $\mathrm{c}$ and exhibited the coexistence of edge-on and face-on structures of DBC where the latter was identical to those without the shear treatment, suggesting that the shear either became ineffective at long distance propagation or was frustrated by the nonuniform evaporation of the solvent. ${ }^{12,13}$ The length of the stripes appeared smaller for solvents with lower boiling points and thus faster evaporation rates. In 1,2,4-trichlorobenzene (bp $213{ }^{\circ} \mathrm{C}$ ) and 1,2-dichrobenzene (bp $178^{\circ} \mathrm{C}$ ), the domain size of DBC became $3 \mathrm{~mm}$ or smaller. In the case of toluene (bp $140{ }^{\circ} \mathrm{C}$ ), careful handling was necessary to obtain a domain size of $1 \mathrm{~mm}$. The influence of solvent evaporation could be precluded by using method 1 . When a cover slide was utilized to suppress solvent evaporation, even for toluene, features of edge-on orientation were imaged on every location.

In conclusion, presented here are STM images with spatial resolution at the molecular level to demonstrate that long-range arrays of edgeon polyaromatics can be assembled predominantly in one single domain. This procedure of a planar Couette-like flow is shown applicable to DBC and other polyaromatics (Supporting Information). Factors such as the flow rate, the steadiness of the flow, the flow direction relative to the unit cell vector of the substrate, and dewetting of solvents are found important to the domain size and the packing quality. Further investigation will be focused on films prepared on substrate suitable for molecular electronics such as glass or silica wafer, which are not conductive for STM imaging and thus are challenging for acquiring molecularly resolved images.

Acknowledgment. The authors thank the National Science Council, Taiwan, for supporting this work (NSC 96-2752-M-002-019PAE). C.-h.C acknowledges the College of Science (NTU) for startup funding (Grant 95R0034-02).

Supporting Information Available: Experimental details and STM images of the polyaromatic films. This material is available free of charge via the Internet at http://pubs.acs.org.

\section{References}

(1) Barth, J. V.; Costantini, G.; Kern, K. Nature 2005, 437, 671.

(2) Grill, L.; Dyer, M.; Lafferentz, L.; Persson, M.; Peters, M. V.; Hecht, S. Nat. Nanotechnol. 2007, 2, 687.

(3) van Hameren, R.; Schön, P.; van Buul, A. M.; Hoogboom, J.; Lazarenko, S. V.; Gerritsen, J. W.; Engelkamp, H.; Christianen, P. C. M.; Heus, H. A.; Maan, J. C.; Rasing, T.; Speller, S.; Rowan, A. E.; Elemans, J. A. A. W.; Nolte, R. J. M. Science 2006, 314, 1433.

(4) Busse, F. H. Science 2004, 305, 1574.

(5) Hoeben, F. J. M.; Jonkheijm, P.; Meijer, E. W.; Schenning, A. P. H. J. Chem. Rev. 2005, 105, 1491.

(6) Wan, Y.; Zhao, D. Y. Chem. Rev. 2007, 107, 2821.

(7) Yoshio, M.; Mukai, T.; Ohno, H.; Kato, T. J. Am. Chem. Soc. 2004, 126, 994.

(8) Li, C.-W.; Wang, C.-I; Liao, H.-Y.; Chaudhuri, R.; Liu, R.-S. J. Org. Chem. 2007, 72, 9203.

(9) Ito, S.; Wehemeier, M.; Brand, D. J.; Kübel, C.; Epsch, R.; Rabe, J. P.; Müllen, K. Chem.-Eur. J. 2000, 4327.

(10) Stabel, A.; Herwig, P.; Müllen, K.; Rabe, J. P. Angew. Chem., Int. Ed. Engl. 1995, 34, 1609.

(11) Utilized to estimate the flow rate was oversaturated dibenzo[ $g, p]$ chrysene in phenyloctane which resulted in very small particles. Upon applying the shear treatment, the particles moved in parallel. By monitoring the particular motion with an optical microscope, the linear flow rate of the solvent was determined on the basis of the assumption that the particles and the solvent had the same velocity.

(12) Palermo, V.; Morelli, S.; Simpson, C.; Müllen, K.; Samorì, P. J. Mater. Chem. 2006, 16, 266.

(13) Lin, Z.; Granick, S. J. Am. Chem. Soc. 2005, 127, 2816.

(14) Hong, S. W.; Xu, J.; Lin, Z. Nano Lett 2006, 6, 2949.

(15) Byun, M.; Hong, S. W.; Zhu, L.; Lin, Z. Langmuir 2008, 24, 3525.

JA8024017 